\title{
Simulation studies of annihilation-photon's polarisation via Compton scattering with the J-PET tomograph
}

\author{
N. Krawczyk ${ }^{1}$ - B. C. Hiesmayr ${ }^{2}$ - J. Chhokar ${ }^{1}$ - C. Curceanu ${ }^{3} \cdot$ E. Czerwiński ${ }^{1}$. \\ K. Dulski ${ }^{1}$ - A. Gajos ${ }^{1}$ - M. Gorgol ${ }^{4}$ - N. Gupta-Sharma ${ }^{1}$ • B. Jasińska ${ }^{4}$ - D. Kisielewska ${ }^{1}$. \\ G. Korcyl ${ }^{1}$ - P. Kowalski ${ }^{5}$ - W. Krzemień ${ }^{6}$. T. Kozik ${ }^{1}$ - E. Kubicz ${ }^{1} \cdot$ M. Mohammed ${ }^{1}$. \\ Sz. Niedźwiecki ${ }^{1}$ - M. Pałka ${ }^{1}$ - M. Pawlik-Niedźwiecka ${ }^{1}$ • L. Raczyński ${ }^{5}$ - J. Raj ${ }^{1}$. \\ K. Rakoczy ${ }^{1}$ - Z. Rudy ${ }^{1}$ - S. Sharma ${ }^{1}$. Shivani ${ }^{1} \cdot$ R. Y. Shopa ${ }^{5} \cdot$ M. Silarski ${ }^{1}$. \\ M. Skurzok ${ }^{1} \cdot$ W. Wiślicki ${ }^{5} \cdot$ B. Zgardzińska ${ }^{4} \cdot$ M. Zieliński $^{1} \cdot$ P. Moskal ${ }^{1}$
}

Published online: 20 August 2019

(C) The Author(s) 2019

\begin{abstract}
J-PET is the first positron-emission tomograph (PET) constructed from plastic scintillators. It was optimized for the detection of photons from electron-positron annihilation. Such photons, having an energy of $511 \mathrm{keV}$, interact with electrons in plastic scintillators predominantly via the Compton effect. Compton scattering is at most probable at an angle orthogonal to the electric field vector of the interacting photon. Thus registration of multiple photon scatterings with J-PET enables to determine the polarization of the annihilation photons. In this contribution we present estimates on the physical limitation in the accuracy of the polarization determination of $511 \mathrm{keV}$ photons with the J-PET detector.
\end{abstract}

Keywords Compton scattering $\cdot \mathrm{J}-\mathrm{PET} \cdot$ Polarization

This article is part of the Topical Collection on Proceedings of the 13th International Conference on Low Energy Antiproton Physics (LEAP 2018) Paris, France, 12-16 March 2018

Edited by Paul Indelicato, Dirk van der Werf and Yves Sacquin

\section{N. Krawczyk}

nikodem.krawczyk@gmail.com

1 Faculty of Physics, Astronomy and Applied Computer Science, Jagiellonian University, S.

Łojasiewicza 11, 30-348 Kraków, Poland

2 Faculty of Physics, University of Vienna, Boltzmanngasse 5, 1090 Vienna, Austria

3 INFN, Laboratori Nazionali di Frascati CP 13, Via E. Fermi 40, 00044, Frascati, Italy

4 Department of Nuclear Methods, Institute of Physics, Maria Curie-Sklodowska University, Pl. M. Curie-Sklodowskiej 1, 20-031 Lublin, Poland

5 Department of Complex Systems, National Centre for Nuclear Research, 05-400 Otwock-Świerk, Poland

6 High Energy Department, National Centre for Nuclear Research, 05-400 Otwock-Świerk, Poland 


\section{Introduction}

J-PET is a multipurpose detector designed for the development of medical imaging [15], for studies of discrete symmetries in decays of positronium atoms [6], as well as for investigations of multipartite quantum entanglement of photons originating from positronium annihilation [7-9]. J-PET is built from 192 plastic scintillator strips arranged axially in three cylindrical layers [10]. The cross section of the detector is shown in the left panel of Fig. 1. Detailed description of the detector can be found in previous publications, here we only briefly outline it's main features. Photons interacting in a scintilators create light signals which are converted to electrical signals by photomultipliers placed at opposite ends of each strip [1]. Position and time of the interaction in the detector material are determined based on the arrival time of light signals at both ends of the scintillator strips. The signals are probed in the voltage domain with the accuracy of about 30 ps by a newly developed type of front-end electronics [11] and the data are collected by the novel trigger-less and reconfigurable data acquisition system $[12,13]$.

Photons from the $e^{+} e^{-}$annihilation interact in plastic scintillators predominantly via the Compton effect and in the J-PET detector a few percent of them undergo secondary scatterings. Events with multiple scatterings may be used to estimate the linear polarization of the initial photon at the moment of its interaction. Taking into account that the scattering is most likely at an angle orthogonal to the polarization we may estimate polarization direction by [6]: $\hat{\epsilon}=\hat{k} \times \hat{k}^{\prime}$ where $\hat{k}$ and $\hat{k}^{\prime}$ denotes the momentum unit vectors of the photon before and after the Compton scattering, respectively. Access to the polarization degree of freedom in the case of measurements of photons from the decays of positronium opens new perspectives for studies of the discrete symmetries $[6,14]$ and multipartite quantum entanglement $[7,15]$. In this article, we discuss the physical limits of the accuracy for the polarization determination of annihilation photons via Compton scattering.

\section{Compton scattering and Klein-Nishina formula}

Angular distribution of a scattered radiation of a linear polarized incoming photon can be described by the Klein-Nishina formula [16, 17]:

$$
\begin{aligned}
\frac{d \sigma(E, \theta, \eta)}{d \Omega} & =\frac{r_{0}^{2}}{2}\left(\frac{E^{\prime}}{E}\right)^{2}\left(\frac{E}{E^{\prime}}+\frac{E^{\prime}}{E}-2 \sin ^{2} \theta \cos ^{2} \eta\right) \\
& =\frac{r_{0}^{2}}{2}\left(\frac{E^{\prime}}{E}\right)^{2}\left(\frac{E}{E^{\prime}}+\frac{E^{\prime}}{E}-\sin ^{2} \theta\right)\{1-\mathcal{V}(\theta, E) \cos (2 \eta)\}
\end{aligned}
$$

with

$$
\begin{aligned}
E^{\prime}(E, \theta) & =\frac{E}{1+\frac{E}{m_{e} c^{2}}(1-\cos \theta)}, \\
\mathcal{V}(\theta, E) & =\frac{\sin ^{2} \theta}{\frac{E}{E^{\prime}}+\frac{E^{\prime}}{E}-\sin ^{2} \theta}
\end{aligned}
$$

where $E$ is the energy of initial photon, $E^{\prime}$ is the energy of photon after scattering, $\theta$ is the Compton scattering angle and $\eta$ is the angle between scattering and polarization planes. The expression $\mathcal{V}(\theta, E)$ quantifies the interference contrast, the a priori visibility [18]. It is a typical quantity showing up in any double-slit-like scenario. Particularly, it has been shown of the Mott scattering, namely Rutherford scattering with identical particles and in 

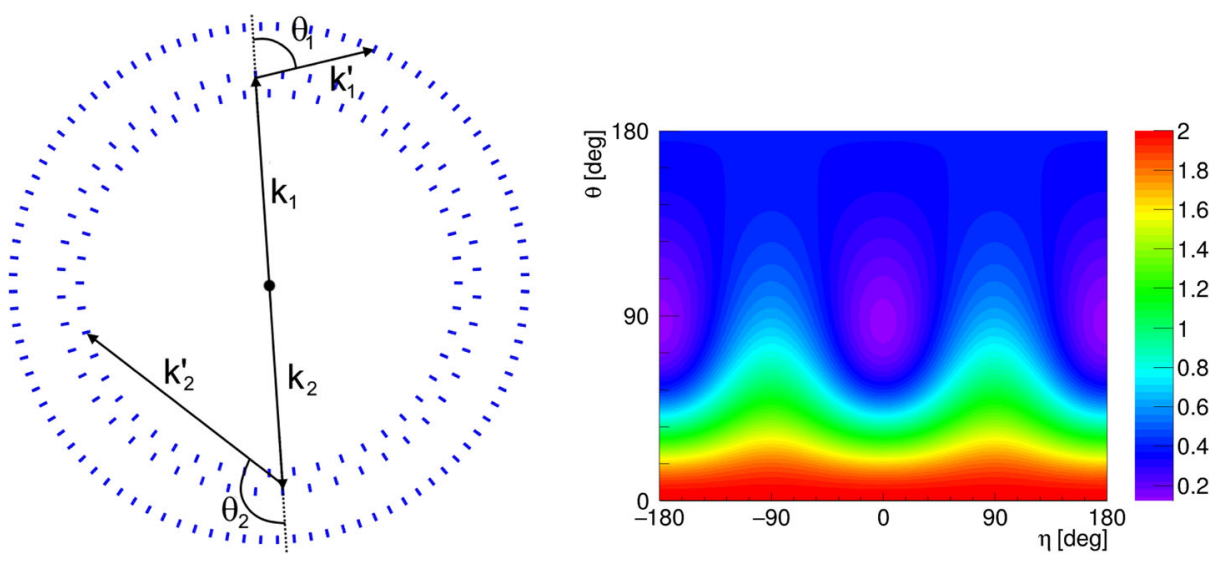

Fig. 1 Left: Cross section of the J-PET detector. Superimposed arrows indicate primary and scattered momenta of photons originating from para-positronium decaying in the center of the detector. Present J-PET tomograph is built from plastic scintillators strips arranged axially in three rings with radii of $425 \mathrm{~mm}, 467.5$ $\mathrm{mm}$ and $575 \mathrm{~mm}$. Right: Klein-Nishina differential cross section (1) as a function of $\eta$ and $\theta$ angles (see text)
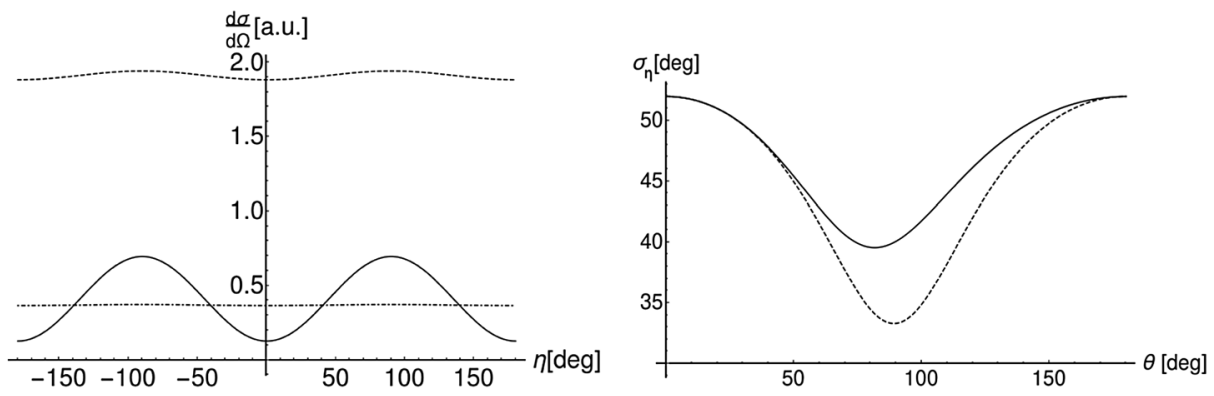

Fig. 2 Left: Klein-Nishina differential cross section (1) for photons with initial energy $E=511 \mathrm{keV}$ presented as a function of angle $\eta$. Dashed-, solid-, and dot-and-dashed-line show results for scattering at angles $\theta=10^{\circ}, \theta=81.67^{\circ}$, and $\theta=170^{\circ}$, respectively. Right: Uncertainty $\left(\sigma_{\eta}\right)$ of the determination of the polarization direction for $511 \mathrm{keV}$ photons (solid line) and $100 \mathrm{keV}$ photons (dashed line) via Compton scattering [6]. For definition of $\theta$ and $\eta$ angles see text

the decay of neutral mesons, which are superpositions of particle and antiparticle states [18]. Obviously, if the visibility is close to zero, the oscillation due to the polarization degree of freedom are not observable. In this case no information on the polarization degree of freedom can be deduced. For $511 \mathrm{keV}$ photons the maximum of the visibility is obtained for a scattering angle of $\theta=81.67^{\circ}$ and the minimum of the visibility is obtained for small and large scattering angles $\theta$, independent of the energy.

The right panel of Fig. 1 shows the calculated double differential cross section for Compton scattering of $511 \mathrm{keV}$ photons, as a function of $\eta$ and $\theta$ angles (1). As expected, for $\theta$ around $82^{\circ}$ a most pronounced modulation of the cross section as a function of the $\eta$ angle is observed. The amplitude of modulations decreases towards higher and lower values of $\theta$. A quantitative comparison of this dependence is shown for three chosen angles in the left panel of Fig. 2. 
The distributions shown in the left panel of Fig. 2 may be interpreted as a resolution function for the determination of the polarization direction by $\hat{\epsilon}$. Due to different values of cross sections for scattering under various Compton angles $\theta$, in order to compare the likelihoods of scattering parallel and normal to polarization, we introduce the following normalization $P(E, \theta, \eta)$ :

$$
P(E, \theta, \eta)=N(E, \theta) \cdot \frac{d \sigma(E, \theta, \eta)}{d \Omega},
$$

where the normalization factor $N(E, \theta)$ is defined as:

$$
N(E, \theta)=1 / \int_{-\pi}^{\pi} \frac{d \sigma(E, \theta, \eta)}{d \Omega} d \eta
$$

which for fixed initial energy $E$ and Compton scattering angle $\theta$ can be understood as the probability density distribution of the angle $\eta$. Therefore we can calculate mean value of such distribution:

$$
\mu=\int_{0^{\circ}}^{180^{\circ}} \eta P(E, \theta, \eta) d \eta
$$

and it's standard deviation

$$
\sigma_{\eta}=\sqrt{\int_{0^{\circ}}^{180^{\circ}}(\eta-\mu)^{2} P(E, \theta, \eta) d \eta},
$$

which would be a measure of how accurate is our assumption that scatterings are always parallel to the polarization vector.

\section{Summary}

The J-PET tomograph built from plastic scintillators enables measurements of the polarization of photons at an event-by-event basis. In this article it was shown that the physical limitations for the accuracy of the estimation of the linear polarization direction of the 511 $\mathrm{keV}$ photons, due to the nature of the Compton scattering, is equal to $40^{\circ}(\sigma)$ for $\theta \approx 82^{\circ}$ and it worsens towards smaller and larger scatterings angles. As a result we have shown that studies of entanglement and discrete symmetries in positronium decays involving polarization should be concentrated for scattering angles around $82^{\circ}$ while for the forward and backward scatterings the information about polarization direction is not attainable.

Acknowledgements The authors acknowledge technical and administrative support of A. Heczko, M. Kajetanowicz and W. Migdał. This work was supported by The Polish National Center for Research and Development through grant

INNOTECH-K1/IN1/64/159174/NCBR/12, the Foundation for Polish Science through the MPD and TEAM/2017-4/39 programmes, the National Science Centre of Poland through grants no. 2016/21/B/ST2/01222, 2017/25/N/NZ1/00861, the Ministry for Science and Higher Education through grants no. 6673/IA/SP/2016, 7150/E-338/SPUB/2017/1 and

7150/E-338/M/2017, and the Austrian Science Fund FWF-P26783.

Open Access This article is distributed under the terms of the Creative Commons Attribution 4.0 International License (http://creativecommons.org/licenses/by/4.0/), which permits unrestricted use, distribution, and reproduction in any medium, provided you give appropriate credit to the original author(s) and the source, provide a link to the Creative Commons license, and indicate if changes were made. 


\section{References}

1. Moskal, P., et al.: Nucl. Instrum. Meth. A 764, 317 (2014)

2. Moskal, P., et al.: Nucl. Instrum. Meth. A 775, 54 (2015)

3. Moskal, P., et al.: Phys. Med. Biol. 61, 2025 (2016)

4. Raczyński, L., et al.: Phys. Med. Biol. 62, 5076 (2017)

5. Moskal, P., et al.: Phys. Med. Biol. 64, 055017 (2019)

6. Moskal, P., et al.: Acta Phys. Polon. B 47, 509 (2016)

7. Hiesmayr, B.C., Moskal, P.: Sci. Rep. 7(1), 15349 (2017)

8. Hiesmayr, B.C., Moskal, P.: Sci. Rep. 9, 8166 (2019)

9. Nowakowski, M., Bedoya Fierro, D.: Acta Phys. Polon. B 48, 1955 (2017)

10. Niedźwiecki, S., et al.: Acta Phys. Polon. B 48, 1567 (2017)

11. Pałka, M., et al.: JINST 12, 08001 (2017)

12. Korcyl, G., et al.: Acta Phys. Pol. 47, 491 (2016)

13. Korcyl, G., et al.: IEEE Trans. Med. Imaging 37, 11 (2018)

14. Acín, A., Latorre, J.I., Pascual, P.: Phys. Rev A 63, 042107 (2001)

15. Spengler, S., et al.: Phys. Rev. A 86, 022311 (2012)

16. Klein, O., Nishina, Y.: Z. Physik 52, 853 (1923)

17. Evans, R.D.: Corpuscles and radiation in matter II / Korpuskeln und Strahlung in Materie II, pp. 218-298. Springer, Berlin (1958)

18. Bramon, A., Garbarino, G., Hiesmayr, B.C.: Phys. Rev. A 69, 022112 (2004)

Publisher's note Springer Nature remains neutral with regard to jurisdictional claims in published maps and institutional affiliations. 